\title{
CONTRIBUIÇÕES DA MONITORIA DE ENSINO NA FORMAÇÃO PROFISSIONAL EM ENFERMAGEM: RELATO DE EXPERIÊNCIA
}

\section{CONTRIBUTIONS OF THE MONITORING OF TEACHING IN PROFESSIONAL NURSING TRAINING: EXPERIENCE \\ Amanda Alcantara de Sousa ${ }^{1}$; Brenda Belém Luna Sampaio ${ }^{1}$; Rachel de Sá Barreto Luna Callou Cruz ; ; Glauberto da Silva Quirino² e Dayanne Rakelly de Oliveira ${ }^{2}$}

Universidade Regional do Cariri, Docente do Departamento de Enfermagem e do Programa de Pós-Graduação em Enfermagem ${ }^{1}$, Universidade Regional do Cariri, Docente do Departamento de Enfermagem e do Programa de PósGraduação em Enfermagem².

\section{Abstract}

This article aims to describe the teaching monitoring experience of the Nursing discipline in the Nursing Care Process of Women linked to the Nursing Course of the Regional University of Cariri (URCA), in the city of Crato, Ceará. An experience report of the process dynamics of organization and development of monitoring activities and their configuration was carried out over two consecutive semesters - first and second semesters of 2018. The motivation for writing the manuscript was through the need to express the reflection that the lived process produced in this specific practice of academic formation. During this period, 20 hours were made available to monitor activities, including practical classes for students in the institution's semiology laboratory, supervised by the teacher, internal training, reviews related to evaluations, and guidelines for students on practical scenarios in maternity and basic health units. Academic monitoring enabled successful experiences for both parties involved. It enabled the monitors to develop skills for the practice of teaching practice, as well as providing students with a moment of information exchange, contributing to the strengthening of the teaching-learning process.

\section{Resumo}

Este artigo tem por objetivo descrever a experiência da monitoria de ensino da disciplina Enfermagem no Processo de Cuidar em Saúde da Mulher vinculada ao Curso de Enfermagem da Universidade Regional do Cariri (URCA), no município de Crato, Ceará. Foi realizado um relato de experiência da dinâmica processual de organização e desenvolvimento das atividades de monitoria e sua configuração ao longo de dois semestres consecutivos - primeiro e segundo semestres de 2018. A motivação pela escrita do manuscrito se deu por meio da necessidade de expressar a reflexão que o processo vivido produziu nessa prática específica de formação acadêmica. Durante esse período, foram disponibilizadas, semanalmente, 20 horas a atividades referentes à monitoria, dentre elas, aulas práticas para os discentes em laboratório de semiologia da instituição, sob supervisão $d a$ docente orientadora, capacitações internas, revisões relacionadas a avaliações, resoluções de simulados e orientações para discentes sobre os cenários práticos nas maternidades e unidades básicas de saúde. A monitoria acadêmica possibilitou experiências exitosas para ambas as partes envolvidas. Oportunizou as monitoras o desenvolvimento de habilidades para o exercício da prática docente, além de proporcionar aos discentes um momento de troca de informações, contribuindo para o fortalecimento do processo ensino-aprendizagem.

Palvras-chave: Enfermagem, Ensino, Aprendizagem. 
A monitoria acadêmica, orientada pela Lei № 9.394/961, consiste em uma ferramenta relevante no ensino superior pela oportunidade de ampliação de experiências ${ }^{2}$, a qual contribui para formação integrada do discente nas atividades de ensino, pesquisa e extensão ${ }^{3}$. Nessa perspectiva, a monitoria constitui-se em um ambiente de aprendizagem, no contexto acadêmico, que estimula a formação docente e colabora na produção de egressos mais qualificados para o mercado de trabalho ${ }^{2}$.

Ademais, a monitoria acadêmica deve pautar-se na realização de atividades que envolvam o estabelecimento de práticas e experiências pedagógicas que fortalecem o aprendizado do monitor, contribuindo para o alcance de um processo ensino-aprendizagem efetivo, com relevante incentivo à busca de conhecimento e a mobilização do senso de responsabilidade pela formação do outro ${ }^{4}$.

Ao atuar como monitor o discente desenvolve habilidades e competências teóricopráticas importantes para o crescimento acadêmico, pessoal e profissional. Além do fortalecimento da relação discente-monitordocente, proporciona também troca de experiências e desenvolvimento da comunicação ${ }^{5}$.

A monitoria na graduação em enfermagem oportuniza ao discente-monitor enxergar uma nova área de atuação além da assistência, oferece uma visão holística sobre como atuar no âmbito de multiplicador de conhecimento, além de introduzi-lo nos métodos didáticos, de suma importância para atuação do enfermeiro na assistência e em diversas áreas ${ }^{6}$.

$\mathrm{Na}$ matriz curricular no curso de enfermagem, a disciplina Enfermagem no Processo de Cuidar em Saúde da Mulher possui uma carga horária teórico-prática que aborda aspectos relativos à saúde da mulher em um contexto biopsicossocial. Abrange as áreas de ginecologia-obstetrícia no âmbito da atenção primária, secundária e terciária, envolvendo o processo saúde-doença e assistência à mulher desde o pré-natal, gestação, parto e puerpério, até ações de planejamento reprodutivo e climatério.

Por apresentar um componente curricular extenso, torna-se relevante a atuação de monitores na disciplina, o que beneficia ambas as partes envolvidas, pois contribui para construção do aprendizado do discente monitorado e do monitor.
Sendo assim, este estudo justifica-se pela necessidade de maior visibilidade à monitoria como um método de ensino-aprendizagem, dentro das necessidades de formação acadêmica, que proporciona através da atuação mútua entre discente-monitor-docente, o desenvolvimento de atividades, que permitem o pensamento críticoreflexivo para a aquisição de habilidades necessárias para a prática em saúde, despertando no discente-monitor o interesse pela docência.

Portanto, este estudo tem como objetivo relatar a experiência da monitoria junto à disciplina Enfermagem no Processo de Cuidar em Saúde da Mulher e sua contribuição para a formação profissional, sob o olhar das monitoras envolvidas no processo.

\section{Metodologia}

Trata-se de um estudo descritivo, do tipo relato de experiência, realizado a partir da vivência discente na monitoria voluntária da disciplina Enfermagem no Processo de Cuidar em Saúde da Mulher, na Universidade Regional do Cariri (URCA). O período de vivência teve duração de fevereiro a dezembro de 2018 , referente aos dois semestres letivos da universidade, com a participação de duas discente-monitoras.

A disciplina Enfermagem no Processo de Cuidar em Saúde da Mulher integra o componente curricular obrigatório do curso de graduação em Enfermagem, no sétimo semestre do curso, com carga horária total de 288 horas, sendo 200 horas de aulas teóricas e 88 horas práticas, distribuídas em atividades vinculadas à Atenção Primária em Saúde (APS) e maternidades. Conta com um total de cinco professores, dois mestres e três doutores.

As estratégias e recursos de ensino utilizados nas aulas teóricas e de laboratório incluem aulas expositivas dialogadas, rodas de conversa, debates em grupos, estudos de caso, filmes e práticas em laboratório. As práticas em campo funcionam a partir da formação de grupos e rodízios desses entre a APS e as maternidades da Região Metropolitana do Cariri, onde os acadêmicos podem desenvolver atividades referentes à assistência à mulher no ciclo gravídico-puerperal, saúde sexual e reprodutiva, prevenção de câncer de colo uterino, controle de câncer de mama e climatério/menopausa.

A monitoria possui carga horária semanal de 20 horas semanais e o seu planejamento baseava-se na elaboração e revisão do cronograma, planejamento das atividades teóricas e práticas, elaboração de materiais 
didáticos a serem utilizados durante os encontros, busca na literatura de temas abordados na disciplina e estudos individuais ou em grupo.

As estratégias utilizadas durante a prática de monitoria envolveram a utilização dos materiais didáticos como instrumentos metodológicos, aulas expositivas-dialogadas, discussão e reflexão de artigos com temáticas pertinentes à disciplina, práticas em laboratório e resolução de questões de residência, além de acompanhamento de atividades de educação em saúde, desenvolvidas pelos discentes para os servidores da universidade, e construção de instrumentos de coleta de dados para mulher em idade reprodutiva e para a gestante.

\section{Resultados e Discussão}

$A$ atividade de monitoria proporcionou as monitoras do estudo crescimento pessoal e profissional, desenvolvimento de competências como a autonomia, responsabilidade, criatividade, aperfeiçoamento da capacidade de liderar e comunicar-se, assim como, o planejamento frente às tarefas relativas à função do monitor, permitindo que estas assumissem um comportamento de docente.

Corroborando com essa experiência, estudos apontam resultados positivos da inserção do discente na monitoria, à medida que fortalece a relação interpessoal, proporciona maior conhecimento em relação à temática da disciplina, permite $o$ desenvolvimento de habilidades no campo de ensino-aprendizagem e o estímulo para a atividade docente ${ }^{7,5}$.

Destaca-se que tanto os monitores como os discentes da disciplina são beneficiados com as atividades de monitoria, uma vez que, propicia atitudes autônomas diante do conhecimento, incentivando-os à responsabilidade e ao compromisso com a própria aprendizagem ${ }^{8}$.

Por terem como compromisso a partilha de conhecimentos teórico-práticos ligados à sua área de atuação na turma, os monitores precisam sentir-se seguros quanto as temáticas abordadas na disciplina, dessa forma, buscam atualizaremse constantemente e aprimorar suas habilidades técnicas, a fim de, esclarecer dúvidas e ter bom desempenho na assistência (ANDRADE et al., 2018).

Nesse sentido, observou-se que a prática da monitoria contribuiu para a desenvoltura das monitoras, condicionando maior segurança em compartilhar seus conhecimentos à medida que foram desenvolvidas as atividades propostas de temáticas de interesse da área de ginecologiaobstetrícia.

Visando uma melhor abordagem nas atividades de monitoria foram utilizadas metodologias ativas que incluíram painéis de circuito de conhecimentos, debates de estudos de caso e artigos, simulados, organização de fluxogramas e práticas no laboratório de enfermagem.

Em seu estudo, Chaves et al. ${ }^{9}$ (2020) apontam que a implantação de metodologias ativas como a gamificação, Aprendizado Baseado em Problemas, estudos de caso, treinamento de habilidades técnicas obteve êxito, pois mostraram-se como estratégias didáticas eficientes que contribuíram para uma aprendizagem significativa.

Percebe-se que existem variadas metodologias que podem ser utilizadas de acordo com as situações, disciplinas, conteúdos e características dos discentes envolvidos. Logo, espera-se que o emprego dessas metodologias seja capaz de romper com o modelo tradicional, por tanto tempo empregado, com possibilidades de formação de um profissional enfermeiro crítico-reflexivo $^{10}$.

Nesse sentido, durante as monitorias identificavam-se o conhecimento prévio dos discentes adquiridos nas aulas e estudos individuais, mediante aplicação das metodologias ativas. A partir desse método foi possível reconhecer os principais tópicos que os discentes apresentavam dificuldades. Em seguida, realizava-se a discussão da temática com base em evidências científicas, sanando as dúvidas apresentadas.

Ao associar a prática da monitoria acadêmica com o desenvolvimento de metodologias ativas de ensino, reforça-se a capacidade de julgamento, o raciocínio crítico e clínico, além de favorecer a tomada de decisão, ao possibilitarem uma discussão transversal, que favorece o ensino com integração de conteúdo, tornando o discente sujeito ativo no seu processo de aprendizagem ${ }^{9}$.

É certo que discentes engajados na graduação, não só nas aulas, mas em atividades de monitoria, grupos de pesquisa e extensão tendem a possuir um perfil profissional diferenciado e a se destacarem no mercado de trabalho, uma vez que, o engajamento para além dos limites da sala de aula torna possível uma formação mais crítica e alinhada às diversas realidades fora da universidade ${ }^{11}$.

Destarte, a vivência da monitoria possibilitou o engajamento em atividades de 
pesquisa e extensão, ampliando o conhecimento nas atividades acadêmicas extracurriculares, refletidas na construção de instrumentos de coletas de dados e a realização de atividades de educação em saúde que abordaram temáticas da disciplina.

A disciplina Enfermagem no Processo de Cuidar em Saúde da Mulher é de grande importância no curso de graduação em enfermagem, visto que, aborda a assistência prestada à mulher em todo o seu ciclo vital, em seu estado saudável e patológico, através de promoção, prevenção e reabilitação da saúde, com o objetivo de desenvolver competências e habilidades para prestar assistência integral à mulher, ao considerar o contexto sociocultural, as questões de gênero, os direitos sexuais e reprodutivos e suas necessidades em saúde.

Assim, dentro das atividades propostas para a monitoria havia a construção de instrumentos de coleta de dados direcionados à mulher, nos diferentes ciclos de vida. Estes instrumentos contemplaram a consulta de prénatal e consulta de enfermagem para mulheres em idade reprodutiva. Os instrumentos elaborados pelas monitoras estão sendo utilizados pelo Núcleo Interdisciplinar de Apoio ao Servidor (NIAS) na URCA durante as consultas.

$O$ instrumento de coleta de dados em enfermagem visa documentar informações de forma objetiva e científica, com possibilidades de estabelecer o perfil do estado de saúde do paciente, identificar diagnósticos e a partir desses planejar as ações de enfermagem necessárias para uma assistência de qualidade ${ }^{12}$. Nesse sentido, a experiência de construir um instrumento de coleta de dados foi de extrema relevância para a formação acadêmica das monitoras.

De igual importância foi desenvolvida pelos discentes, sob orientação das monitoras e docentes, atividade de educação em saúde junto aos servidores da universidade. Quando a monitoria permite realização de atividades práticas junto à comunidade, no contexto da saúde, ela potencializa o empoderamento dos usuários, dos discentes e monitores, que reconstrói conceitos e ressignifica o cuidado em saúde, na lógica da participação popular, permitindo aos monitores e discentes a reflexão das ações de educação em saúde e seu impacto para transformação da realidade ${ }^{13}$.

A experiência com a monitoria traz para as discentes grandes contribuições para sua formação acadêmica, entretanto, o monitor pode enfrentar alguns desafios durante seu desenvolvimento. A monitoria requer o uso de materiais para desenvolver atividades práticas em laboratório que algumas vezes não estavam disponíveis, sendo esse um desafio para o monitor, entretanto nenhuma das atividades propostas no cronograma foi cancelada por esse motivo.

Um outro obstáculo para as atividades de monitoria diz respeito à disponibilidade de tempo, tanto para os discentes como para os monitores, principalmente ao considerar o tempo integral do curso de enfermagem da universidade, o que demandou de ambas as partes pactuações quanto aos dias disponíveis para as atividades.

Ao final de cada semestre letivo foram solicitadas avaliações em relação ao papel do monitor e suas contribuições. Resultados positivos foram identificados a partir dos relatos de discentes que consideraram a monitoria como uma atividade essencial aos processos de aprendizagem, à medida que foram propostas atividades bastante interativas, as quais estimularam a revisão dos conteúdos já estudados e reflexão de alguns temas não abordados em aula. Os discentes apresentaramse satisfeitos quanto às metodologias utilizadas pelo monitor, bem como a sua interação e estabelecimento de vínculos.

Estes dados condizem com estudo realizado com discentes do curso de enfermagem que avaliaram positivamente a criatividade dos monitores, fluência verbal e clareza, pontualidade e assiduidade, relacionamento com os discentes monitorados, segurança e domínio dos monitores na administração das aulas e contribuição da monitoria para a vida do profissional enfermeiro $^{14}$.

\section{Conclusão}

A monitoria acadêmica mostrou-se de grande importância para o monitor, à medida que este adquiriu experiências exitosas, oportunizou o desenvolvimento de conhecimento, autonomia, disciplina e capacidade de trabalhar em equipe, competências muito valorizadas para o mercado de trabalho. Esta prática proporcionou ainda a obtenção de horas complementares necessárias para a formação e constituiu-se como a primeira etapa para a docência, preparando o discente para uma possível prática docente.

Para os discentes da disciplina, a monitoria representou um meio factível e efetivo para sanar dúvidas e maximizar o aprendizado, considerando assim, todas as contribuições para formação acadêmica, dentro do contexto do 
ensino superior. Os docentes foram beneficiados por meio das atividades, posto que o monitor reforçou todos os conteúdos dados em sala de aula, auxiliando-o no melhor desempenho dos discentes.

A monitoria pôde ainda contribuir como um apoio para melhoria da qualidade do ensino na universidade, uma vez que, os monitores auxiliaram no processo ensino-aprendizagem de forma mais dinamizada, de acordo com as principais demandas apresentadas pelos discentes sobre temáticas direcionadas a saúde da mulher.

No entanto, algumas limitações foram identificadas quanto ao exercício da monitoria, como a disponibilidade de tempo e a falta de materiais que subsidiam a prática docente, possibilitando ao monitor o reconhecimento das dificuldades enfrentadas cotidianamente pelo docente nas instituições públicas de ensino superior.

Entende-se que apesar das limitações no desenvolvimento das atividades de monitoria, essa estratégia facilitou a troca de conhecimentos entre discentes e docentes, tornando os discentes aptos a desenvolverem as práticas em saúde.

Deve-se levar em consideração a avaliação final dos discentes em relação as atividades propostas pelos monitores e 0 aprendizado que estas possibilitaram. Essa avaliação possibilita identificar os principais pontos positivos da monitoria para a consolidação do conhecimento dos discentes e reformular pontos passíveis de mudanças.

Sendo assim, sugere-se que novos estudos sejam realizados no intuito de aprofundar os benefícios da monitoria acadêmica para o processo ensino-aprendizagem, principalmente voltados à avaliação qualitativa relacionada à satisfação dos discentes.

\section{Referências}

1. BRASIL. Presidência da República. Casa Civil. Subchefia para Assuntos Jurídicos. Lei no 9394, 20 de dezembro de 1996. Estabelece as diretrizes e bases da educação nacional. Brasília, 20 de dezembro de 1996.

2. PINTO MB, MEDEIROS CSA, ANDRADE LDF, SANTOS NCCB, ALBUQUERQUE AM, RAMALHO MNA. Monitoria Acadêmica: importância e contribuição para a formação do enfermeiro. Rev enferm UFPE on line. 2016; 10(6):1990-7.

3. FERNANDES DCA, FERNANDES HMA, BARBOSA ES, CHAVES MJC, NÓBREGA-THERRIEN
SM. Contribuições da monitoria acadêmica na formação do aluno-monitor do curso de enfermagem: relato de experiência. Debates em educação. 2020; 12(27).

4. ANDRADE EGR, RODRIGUES ILA, NOGUEIRA LMV, SOUZA DF. Contribuição da monitoria acadêmica para o processo ensinoaprendizagem na graduação em enfermagem. Rev Bras Enferm [Internet]. 2018; 71(suppl 4):1690-8.

5. BURGOS CN, BARICATI CCA, MARTINS JT, SCHOLZE AR, GALDINO MJQ, KARINO ME. Monitoria acadêmica na percepção dos estudantes de enfermagem. Rev. Enferm. UFSM. 2019; 9(e37):1-14.

6. NASCIMENTO JT, CARDOSO LTS, ARAÚJO LCN, OLIVEIRA VVN, SILVA ES, SILVA PJTG et al. Monitoria como espaço de iniciação à docência. REAS. 2021; 13(2).

7. TAVARES JS, OLIVEIRA FR, MAIA CMAFG, RODRIGUES WFG. Contribuições da monitoria de anatomia humana na formação acadêmica de estudantes de enfermagem: relato de experiência. Rev enferm UFPE on line. 2017; 11(8): 3176-9.

8. FRISON LMB. Monitoria: uma modalidade de ensino que potencializa a aprendizagem colaborativa e autorregulada. ProPosições. 2016; 27(79): 133-53.

9. CHAVES USB, MARTINS AS, COSTA CCP, BISAGNI C, VIEIRA MLC, JESUS PBR. Relato de experiência da utilização de metodologias ativas na prática da monitoria de um curso de Enfermagem. Research, Society and Development. 2020; 9(9).

10. FONTES FLL, BEZERRA AMFA, SILVA HLL, SANTO IMBE, MARQUES TMC, MORAIS MJA et al. Utilização de metodologias ativas no curso de graduação em enfermagem: uma oportunidade de superação do modelo de ensino tradicional. Research, Society and Development. 2021; 10(1):1-10.

11. CORTEZ TRP. O indissociável tripé ensino, pesquisa e extensão na formação do profissional jurista apto a atuar nas demandas sociais. Revista Manus luris. 2020; 1(1):43-49.

12. TAVARES FMM, TAVARES WS. Elaboração de um instrumento de sistematização da assistência de enfermagem: relato de experiência. Revista de Enfermagem do CentroOeste Mineiro. 2018; 8(esp.):1-8.

13. BARBOSA LBM, GOULART BF, BRACARENSE CF, REZENDE MP, VICENTE NG, SIMÕES ALA. ᄀA monitoria de educação em saúde na enfermagem: relato de experiência. Rev enferm UFPE on line. 2017; 11(supl. 7):2979-84.

14. SOUZA JCO, LIMA NR, SILVA JUNIOR RR, 
LIMA NR, LIMA AJG, OLIVEIRA MRS et al. Impactos da monitoria acadêmica de anatomia Humana: Concepções de estudantes de enfermagem. Revista Enfermagem Atual In Derme. 2020; 94(32).

\section{Endereço para Correspondência}

Rachel de Sá Barreto Luna Calhau Cruz

Rua Cel. Antônio Luiz, 1161-bPimenta

Crato - Ceará

CEP: $63105-000$

Tel: (81) 99827-6567

Recebido em 08/04/2019

Aprovado em 07/04/2021

Publicado em 10/05/2021 